\title{
Dedicated sub 0.1 mSv 3DCT using MBIR in children with suspected craniosynostosis: quality assessment
}

\author{
Caroline W. Ernst $^{1}$ • Tine L. Hulstaert ${ }^{1} \cdot$ Dries Belsack $^{1} \cdot$ Nico Buls $^{1}$ • \\ Gert Van Gompel $^{1} \cdot$ Koenraad H. Nieboer ${ }^{1} \cdot$ Ronald Buyl $^{2} \cdot$ Filip Verhelle $^{1}$. \\ Michel De Maeseneer ${ }^{1} \cdot$ Johan de $_{\text {Mey }}{ }^{1}$
}

Received: 10 November 2014 /Revised: 17 April 2015 / Accepted: 1 June 2015 / Published online: 30 June 2015

(C) The Author(s) 2015. This article is published with open access at Springerlink.com

\begin{abstract}
Objective To retrospectively compare image quality of a lowered dose CT protocol to a standard CT protocol in children with suspicion of craniosynostosis.

Methods Forty-eight patients (age 0- 35 months), who presented with a cranial deformity underwent cranial 3D CT to assess sutural patency: between $2009-2010,24$ patients were imaged with a standard protocol (CTDIvol $32.18 \mathrm{mGy}$ ), from 2011-2012, 24 underwent a low dose protocol (0.94 mGy) combined with iterative reconstruction. Image quality was evaluated by both expert reading and objective analysis. Differences were assessed by independent t-test and Mann-Whitney U test, interreader agreement by Cohen's Kappa test.

Results Effective dose of the low dose protocol was $0.08 \mathrm{mSv}$, corresponding to a reduction of $97 \%$. Image quality was similar in both groups in terms of overall diagnostic acceptability, objective noise measurements, subjective cranial bone edge sharpness and presence of artefacts. For objective sharpness of cranial bone-brain interface and subjective perception of noise, the images of the low dose protocol were superior. For all evaluated structures, interreader agreement was moderate to almost perfect.

Conclusion In the diagnosis of craniosynostosis in children with cranial deformities, a dedicated sub $0.1 \mathrm{mSv}$ cranial 3DCT protocol can be used without loss in image quality.
\end{abstract}

Caroline W. Ernst

caroline.ernst@uzbrussel.be

1 Department of Radiology, Universitair Ziekenhuis Brussel, Laarbeeklaan 101, 1090 Brussels, Belgium

2 Department of Biomedical Statistics and Informatics, Vrije Universiteit Brussel, Laarbeeklaan 103, 1090 Brussels, Belgium
Key Points

- 3DCT is used for the diagnosis of craniosynostosis.

- Imaging protocols should be optimized to minimize radiation exposure to children.

- Combining $80 \mathrm{kVp}$ with iterative reconstruction can help to reduce dose.

- A sub 0.1 mSv cranial 3DCT protocol can be used without loss of diagnostic quality.

Keywords Multidetector Computed Tomography · Iterative reconstruction $\cdot$ Infant $\cdot$ Radiation protection .

Craniosynostosis
Abbreviations
ASIR Adaptive Statistical Iterative Reconstruction
CT Computed Tomography
CTDI Computed Tomography Dose Index is the current standard for CT dosimetry
CTDI weighted average measurement in a reference
vol phantom divided by the CT pitch factor CTDIvol is used to estimate the relative dose for an exam. It is expressed in milliGrays.
DLP Dose Length Product : product of the CTDIvol and the scan length. It is expressed in milliGray * centimetres
ED Effective Dose measured in Sieverts
FBP Filtered Back Projection the standard used recon- struction technique in CT.
HU Hounsfield Units unit of signal measurements
IR Iterative Reconstruction
IN image noise
IQ image quality
$\mathrm{mSv} \quad$ milliSievert unit of effective dose
MBIR Model Based iterative reconstruction
MDCT MultiDectector Computed Tomography 
SD Standard Deviation

SNR signal to noise ratio

\section{Introduction}

Craniosynostosis is the premature fusion of one or more cranial sutures. It occurs in 3.1 to 4.8 per 10,000 live births and results in cranial deformity and sometimes function impairment due to increased intracranial pressure and restricted brain growth. Early clinical recognition and accurate diagnostic confirmation is essential to ensure proper management of this condition. Imaging studies have traditionally played an important role in the diagnosis and characterization of craniosynostoses. Patency of the major sutures is currently evaluated by three-dimensional Computed Tomography (3DCT). This technique is essential for accurate diagnosis, therapy planning (conservative or surgical), and postoperative follow-up [1-3]. Given that children are two to ten times more radiosensitive than adults, the application of the "as low as reasonably achievable "(ALARA) principle in CT is essential $[1,4-11]$.

To reduce radiation dose in children, several strategies and techniques are currently available, including iterative reconstruction techniques [6, 11-13].

The introduction of a novel iterative reconstruction technique, Model Based Iterative reconstruction (MBIR), in our institution allowed us to develop an optimized low-dose CT protocol.

By modelling the complete optics and noise statistics of the CT system, this technique reduces image noise considerably and improves image quality in terms of spatial resolution and artefacts [13-19]. Applying MBIR technique, we lowered radiation dose while maintaining image quality. This was accomplished by lowering both tube potential and current to the lowest settings currently possible.

The aim of this study was to retrospectively compare the image quality of this protocol to that of the standard protocol in children with a suspicion of craniosynostosis.

\section{Materials and methods}

This retrospective study was approved by our institutional medical ethics board; informed consent was waived. Inclusion criteria were all children who suffered from isolated nonsyndromic cranial deformity, with an age between 0 and 35 months, and referral for cranial 3DCT.

\section{CT protocols and image reconstruction}

From August 2009 to December 2010, 24 consecutive patients underwent a standard cranial 3DCT protocol (which we further designate protocol $\mathrm{A}$ ) on a Philips system (Brilliance CT 64, Philips, Best, The Netherlands). The acquisition parameters were as follows: collimation, $16 \times 0.625 \mathrm{~mm}$; rotation time, $0.5 \mathrm{~s}$ ; tube voltage, $120 \mathrm{kVp}$; fixed tube current of $276 \mathrm{~mA}$; pitch factor, 0.69 , filter type $\mathrm{D}$, and convolution kernel D. Raw data were reconstructed with Filtered Back Projection (FBP).

From August 2011 to December 2012, 24 other consecutive patients, were imaged using an investigative protocol (which we further designate protocol B) on a 64-row detector MDCT (Discovery 750HD, GE Healthcare, Milwaukee, WI). The acquisition parameters were: collimation , $32 \times 0.625 \mathrm{~mm}$; rotation time, $0.8 \mathrm{sec}$; tube voltage, $80 \mathrm{kVp}$; fixed tube current of $10 \mathrm{~mA}$; pitch factor, 0.53; head filter; and the best fitting reconstructed field of view (FOV) for every patient. The raw data were reconstructed by using MBIR technique. (VEO ${ }^{\circledR}, \mathrm{GE}$ Healthcare; Milwaukee, WI).

All patients were imaged from vertex to skull base with the best fitting reconstructed FOV according to the patient's anatomy.

\section{Radiation dose assessment}

CTDIvol and DLP (for a $16 \mathrm{~cm}$ phantom) were recorded for every $\mathrm{CT}$ examination. An estimate of the effective dose (ED) was based on the details of the scan protocol and obtained by $\mathrm{CT}$ dosimetry software using the organ weighting factors of ICRP 103 (Impact CT dosimetry calculator 1.04, impact, St George Healthcare, NHS, London, UK).

\section{Subjective image quality}

Subjective image quality was independently evaluated by two board-certified radiologists (C.E. and D.B. with respectively 15 and 6 years of experience). Prior to this evaluation, ten cases (not included in the study) were evaluated by consensus agreement to improve conformity between the readers. After anonymization, the studies were evaluated by the readers in a blinded fashion and random order. The dose-related data were not available for this reading. The images were evaluated on a PACS system (Impax 6.4.0, $\mathrm{Agfa}^{\circledR}$ HealthCare, Mortsel, Belgium) on a 3 megapixel viewing station at bone window settings (window width, $1500 \mathrm{HU}$; window level, $500 \mathrm{HU}$ ). To assess image quality, all images were reviewed at a slice thickness of $1 \mathrm{~mm}$.

Subjective image quality was scored on a 4 or 5 point scale as described in Table $1[1,20,21]$. Parameters that were assessed included image noise, image sharpness, overall diagnostic acceptability, and artefacts. 
Table 1 Scoring list of subjective image quality

\begin{tabular}{|c|c|c|c|c|}
\hline Score & Image noise & Image sharpness & Overall Diagnostic acceptability & Artifacts \\
\hline 1 & No noise/very low & Structures are well defined with sharp contours & Excellent & No artifacts \\
\hline 2 & Low (normal) & $\begin{array}{l}\text { Contours are not fully sharp, but structures } \\
\text { are defined }\end{array}$ & Good & Minor artifacts \\
\hline 3 & $\begin{array}{l}\text { Considerable with preserved } \\
\text { diagnostic image quality }\end{array}$ & $\begin{array}{l}\text { Structures can be seen, but contours are } \\
\text { barely sharp enough }\end{array}$ & Suboptimal, but still diagnostic & Moderate artifacts \\
\hline 4 & $\begin{array}{l}\text { High, causing nondiagnostic } \\
\text { image quality }\end{array}$ & $\begin{array}{l}\text { Although structures can be visualized, } \\
\text { contours are blurred and images are } \\
\text { insufficient for diagnostic reporting }\end{array}$ & Unacceptable and nondiagnostic & $\begin{array}{l}\text { Major artifacts but } \\
\text { interpretable }\end{array}$ \\
\hline 5 & & Structures cannot be identified & & $\begin{array}{l}\text { Artifacts make image } \\
\text { interpretation impossible }\end{array}$ \\
\hline
\end{tabular}

The edges of the cranial bones were evaluated to grade image sharpness. To determine image sharpness, the inner ear structures and the medial orbital margin were also assessed. However, these structures are not important in the diagnosis of craniosynostosis. The overall diagnostic acceptability was graded both for axial and 3D reconstructions.

Grades for subjective image quality were averaged for both readers before further analysis was performed.

\section{Objective image quality- quantitative measurements}

The signal-to-noise ratio (SNR) at the level of the clivus and sharpness of the cranial bone-brain interface were assessed.

Circular Regions Of Interest (ROIs) with a size of 10 to $15 \mathrm{~mm}^{2}$ were placed on the clivus to obtain a homogenous area. CT numbers (HU values) and noise (standard deviation $\mathrm{SD}$ ) were measured and SNR was calculated by dividing the $\mathrm{CT}$ number by the noise.

To determine the sharpness of the bone-brain interface, in every patient a CT value profile was obtained in image $\mathrm{J}$ (National Institutes of Health, USA) along a straight line. This line was perpendicular to the interface at the lateral margin of the left orbit.

Sharpness was based on the slope of this profile, as shown in Fig. 1.

Fig. 1 Sharpness of bone-brain interface. The sharpness of the interface was based on the slope of the transition curve between $15 \%$ and $80 \%$ of maximum CT number $(\mathrm{HU})$ with bone being the maximal and brain the minimal CT number

\section{Statistical analysis}

Statistical analysis was performed using SPSS ${ }^{\circledR}$ (Version 19.0 for Windows, SPSS, Chicago,). Normal distribution and homogeneity of variance were tested using the Shapiro-Wilk's W test and Levene's test, respectively. Independent samples $t$ tests were used to compare continuous and normally distributed variables such as SNR, sharpness, patient characteristics and radiation dose. Gender was compared using a chi-square test. A non-parametric (Mann-Whitney-U) test was used to analyze discrete variables such as the results obtained with the subjective image quality assessment. This was also done when the continuous variables were not normally distributed. A significance threshold of $p$-value $<0.05$ was used. Interreader agreement was assessed with Cohen's Kappa test and interpreted according to Landis and Koch (1977). When kappa was $<0$, this indicated poor agreement; slight agreement if $0.0-0.20$; fair agreement if 0.21-0.40; moderate agreement if 0.41 0.60 ; substantial agreement if $0.61-0.80$, and almost perfect agreement if 0.81-1 [22].

\section{Results}

Cranial 3DCT was performed in 48 consecutive patients (Table 2). Table 3 shows the distribution of pathology for a

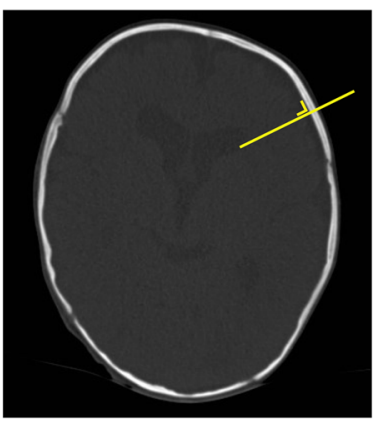

b

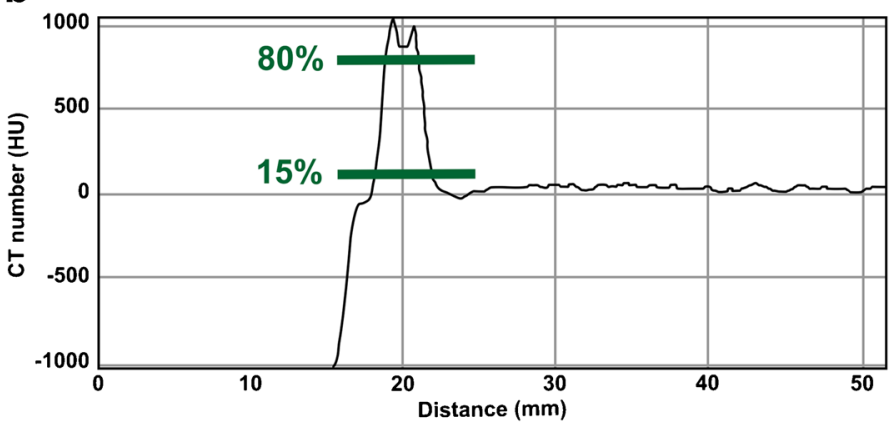


Table 2 Patient characteristics and radiation dose. Mean \pm SD is given for age, DLP, CTDI and ED. A median for most results is given between rounded brackets and age range between square brackets. Statistical analysis was performed using independent $\mathrm{T}$ test and Chi-square test (gender). $P$-values are listed. If results are significant, they are indicated by *

\begin{tabular}{llll}
\hline & Protocol A & Protocol B & P- value \\
\hline Age (months) & $7.3 \pm 4.8[2-24]$ & $8.1 \pm 7.3[0-35]$ & 0.626 \\
Gender (male/female) & $15 / 9(62.5 \% / 37.5 \%)$ & $17 / 7(71 \% / 29 \%)$ & 0.759 \\
DFOV (mm) & $175 \pm 23(171)$ & $177 \pm 24(179)$ & 0.779 \\
CTDIvol (mGy) & $32.18 \pm 0.04(32.19)$ & $0.94 \pm 0.01(0.93)$ & $<0.001^{*}$ \\
DLP (mGy*cm) & $487.48 \pm 33.14(486.70)$ & $15.04 \pm 2.25(14.28)$ & $<0.001^{*}$ \\
Effective dose (mSv) & $3.07 \pm 0.16(3.13)$ & $0.08 \pm 0.00(0.08)$ & $<0.001^{*}$ \\
\hline
\end{tabular}

group A and B separately and the total for both groups. Fortyfour percent of the children were diagnosed with postural plagiocephaly, $47.9 \%$ with craniosynostosis, and $6.2 \%$ with a fracture/calcified cephalic hematoma. In $2.1 \%$ bone structures were normal.

\section{Patient characteristics and radiation dose}

Both groups were similar with regard to age, gender, and displayed field of view (DFOV).

The radiation dose was reduced by approximately $97 \%$ : CTDIvol of protocol B was $2.9 \%$ of CTDIvol of protocol A, DLP $3.1 \%$, and estimated effective dose $2.6 \%$ (Table 2 ).

\section{Subjective image quality}

Figure 2 provides an illustration of images of protocols $\mathrm{A}$ and B. Table 4 shows the results of the image quality assessment.

Overall diagnostic acceptability for axial and 3D images ("excellent") was similar for both protocols. A 'lego' effect was noted on the $3 \mathrm{D}$ images of protocol B without affecting the diagnostic acceptability [23].

A lower amount of noise was perceived for protocol B ("no/very low image noise") compared to protocol A ("normal /low image noise").
Sharpness of cranial bone edges was similar for both groups ("well defined with sharp contours"). Sharpness of inner ear structures and medial orbital margin was better for protocol A than B.

There was no difference in amount and impact of image artefacts ("minor artefacts"). The type of artefacts was different, however. Beam hardening artefacts were present in both groups, but staircase artefacts were only seen in group B. Staircase artefacts (17) were more pronounced along the inferior border of the orbit and at the interface between soft tissue and bone as shown in Fig. 3.

Interobserver agreement (Table 5) varied from substantial to almost perfect, depending on the structures and criteria that were evaluated.

\section{Objective image quality}

In one patient, bony structures were too small for a meaningful measurement, and this patient was excluded from analysis.

In terms of noise no significant difference was seen between protocols A and B. Protocol B resulted in higher CT numbers leading to a higher SNR (Fig. 4, Table 6).

The mean slope of the edge profile between bone and brain parenchyma was $-923.0 \pm 175.8$ in protocol $\mathrm{A}$ and $-1592.3 \pm$ 364.3 in protocol $\mathrm{B}(P=<0.001)$ as illustrated in Fig. 4.
Table 3 Distribution of pathology for group A and B separately and the total for both groups

\begin{tabular}{llll}
\hline Type of pathology & Group A & Group B & Total \\
\hline No pathology & 0 & $1(4.2 \%)$ & $1(2.1 \%)$ \\
Bilamdoid synostosis & 0 & $1(4.2 \%)$ & $1(2.1 \%)$ \\
Unilambdoid synostosis & 0 & 0 & 0 \\
Sagittal synostosis & 0 & $2(8.3 \%)$ & $2(4.2 \%)$ \\
Unicoronal synostosis & $2(8.3 \%)$ & $3(12.5 \%)$ & $5(10.4 \%)$ \\
Bicoronal synostosis & 0 & 0 & 0 \\
Metopic synostosis & $6(25 \%)$ & $9(37.5 \%)$ & $15(31.2 \%)$ \\
Fracture/ calcified cefalhematoma & $2(8 . \%)$ & $1(2.1 \%)$ & $3(6.2 \%)$ \\
Plagiocephaly & $14(58.3 \%)$ & $7(29 \%)$ & $21(43.8 \%)$ \\
\hline
\end{tabular}


Fig. 2 A and $\mathrm{C}$ show

respectively a protocol A axial plane and a 3D image in a patient suffering from right sided coronal craniosynostosis. B and D show respectively a protocol $\mathrm{B}$ axial plane and a 3D image in a patient suffering from left sided coronal craniosynostosis
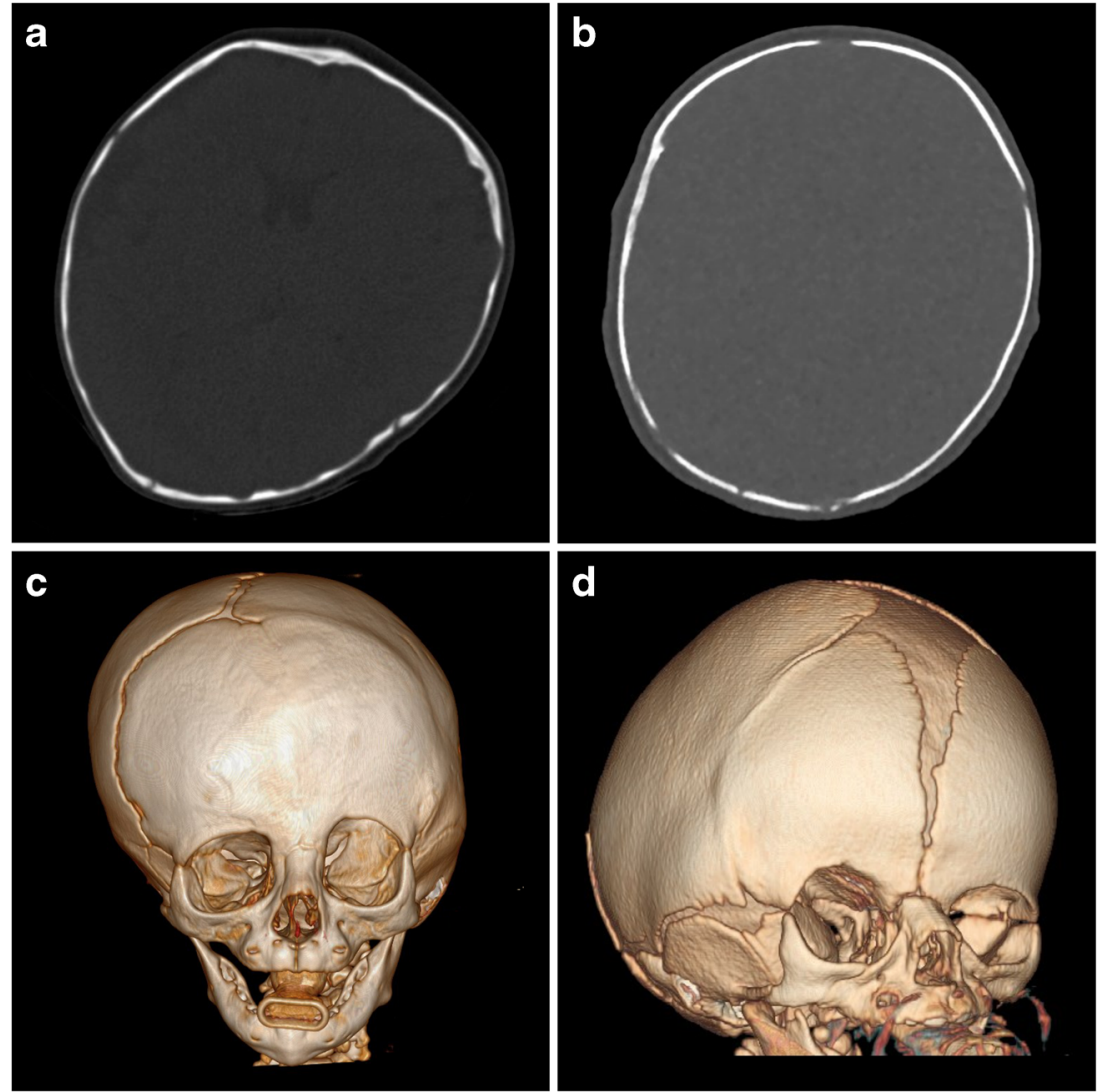

\section{Discussion}

Diagnostic reference levels (DRLs) are a practical tool to promote the assessment of existing scan protocols and appropriate development of new and improved protocols by facilitating the comparison of doses. For adult head CT, recommended reference levels in Europe vary between 60 and $75 \mathrm{mGy}$ [24]; for children, European reference levels are not available now but soon to be expected within the Pi DRL project of the

Table 4 Results of subjective image quality assessment, statistically analyzed by the Mann Whitney $U$ test Median with range between square brackets . If results are significant, they are indicated by *

\begin{tabular}{|c|c|c|c|}
\hline Criteria & Protocol A & Protocol B & $P$ \\
\hline $\begin{array}{l}\text { Overall diagnostic acceptability } \\
\text { (axial images) }(1-4)\end{array}$ & $1[1,2]$ & $1[1,2]$ & 0.161 \\
\hline $\begin{array}{l}\text { Overall diagnostic acceptability } \\
\text { 3D reconstruction }(1-4)\end{array}$ & $1.0[1.0-1.0]$ & $1.0[1.0-1.0]$ & 1 \\
\hline Noise $(1-4)$ & $2.0[2.0-2.0]$ & $1.0[1.0-1.0]$ & $<0.001 *$ \\
\hline $\begin{array}{l}\text { Sharpness of cranial bone edge } \\
(1-5)\end{array}$ & $1.0[1.0-2.0]$ & $1.0[1.0-2.0]$ & 0.161 \\
\hline $\begin{array}{l}\text { Sharpness of inner ear structures } \\
(1-5)\end{array}$ & $2.0[1.0-2.0]$ & $2.0[1.0-3.0]$ & $0.004 *$ \\
\hline $\begin{array}{l}\text { Sharpness of medial orbital } \\
\text { margin }(1-5)\end{array}$ & $1.0[1.0-2.0]$ & $2.0[1.0-2.0]$ & $0.002 *$ \\
\hline Artifacts $(1-5)$ & $2.0[2.0-2.0]$ & $2.0[2.0-2.0]$ & 1 \\
\hline
\end{tabular}

European Society of Radiology [25]. Some previous studies investigated the impact of dose reduction on image quality for specific 3DCT protocols used in the setting of craniosynostosis. Vasquez et al., optimized dose with CTDIvol from $18.8 \mathrm{mGy}$ to $2.3 \mathrm{mGy}$ by using a combination of $80 \mathrm{kV}$ and

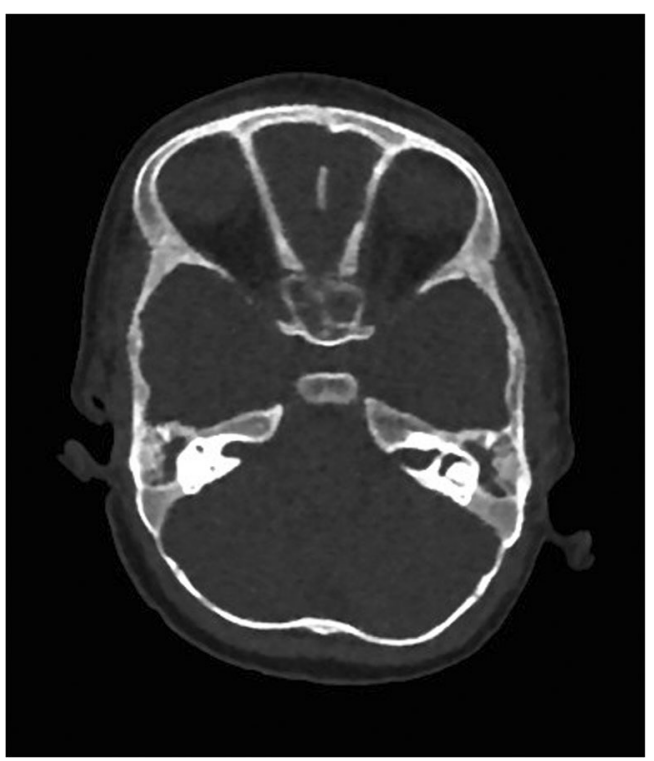

Fig. 3 Protocol B CT image. Presence of staircase artefacts at the frontal and right temporal bone and at the medial orbital margin. The artefacts can be seen at the bone-soft tissue interface 
Table 5 Interobserver agreement of all evaluated criteria for protocol A and B, assessed with the Cohen's Kappa test

\begin{tabular}{lll}
\hline Criteria & Protocol A & Protocol B \\
\hline Noise & NA & NA \\
Sharpness of inner ear structures & 0.75 & 0,84 \\
Sharpness of cranial bone edge & 1.00 & 1.00 \\
Sharpness of medial orbital margin & 0.86 & 0.67 \\
Overall diagnostic acceptability axial images & 1.00 & 1.00 \\
Overall diagnostic acceptability 3D & NA & NA \\
artifacts & NA & NA \\
\hline
\end{tabular}

$<0$ : poor agreement; $0.0-0.20$ : slight agreement; $0.21-0.40$ : fair agreement; 0.41-0.60: moderate agreement; 0.61

0.80:substantial agreement; 0.81-1: almost perfect agreement; NA: not available due to lack of intrareader variance

increased noise index setting. Iterative reconstruction was not applied [1]. Khanna et al., halved the dose of their $120 \mathrm{kV}$ adult scan protocol down to a CTDIvol of $31.25 \mathrm{mGy}$ by only adjusting the tube current [2]. Badve et al., reported a half and quarter dose strategy with a $120 \mathrm{kV}$ protocol at CTDIvol's of, respectively. $11.36 \mathrm{mGy}$ and $5.86 \mathrm{mGy}$, by also reducing only tube current [3]. Applegate et al., reported one case example scanned at $80 \mathrm{kV}$ with CTDIvol of $1.98 \mathrm{mGy}$ [26]. The difference of our scan protocol compared to these reported data is that we included iterative reconstruction in order to achieve further dose reductions. By combining $80 \mathrm{kV}$ with IR, we achieved a median CTDIvol of only $0.94 \mathrm{mGy}$ ( $97 \%$ dose reduction compared to our standard protocol) without
Table 6 Objective image quality assessment of protocol A and B in the clivus analyzed by independent samples $\mathrm{T}$ tests. Mean $\pm \mathrm{SD}$ is mentioned for the bony structures. Significant $P$-values are indicated by $*$ and the group that is in favor is mentioned between, ()

\begin{tabular}{llccl}
\hline Structure & & Protocol A & Protocol B & $P$-value \\
\hline Clivus & CT numbers (HU) & $360.6 \pm 66.5$ & $543.1 \pm 70.4$ & $<0.001^{*}$ \\
& noise (HU) & $36.9 \pm 9.6$ & $34.9 \pm 15.2$ & 0.600 \\
& SNR & $10.3 \pm 2.9$ & $18.3 \pm 7,8$ & $<0.001^{*}$ \\
\hline
\end{tabular}

compromising image quality. To our knowledge, this is the lowest dose ever reported in a study of head CT performed for the investigation of cranial deformation. Also, we are the first institution that reported the use of the MBIR technique in this setting.

Subjective overall diagnostic acceptability is clinically the most important factor of image quality. We found that, even at this very low dose of $0.08 \mathrm{mSv}$, the diagnostic acceptability was excellent and not different from the standard protocol.

For image noise, a difference was found between objective and subjective evaluation. Although no difference in measured noise (SD) was present, the images of protocol B were perceived to be less noisy ("no/very low image noise" vs. "normal /low image noise"). Besides their impact on standard deviation of noise, MBIR algorithms also have an impact on image texture. We speculate that this altered texture, typically described as "plastic, pixilated blotchy appearance", has an impact on the perception of noise $[14,15]$.
Fig. 4 Mean slope of the edge profile between bone and brain parenchyma. The mean slope, defined as $\Delta y / \Delta x$, of protocol $B$ is higher than in protocol $\mathrm{A}$, meaning a sharper edge. Note the higher $\mathrm{CT}$ number (HU) of bony structures in protocol $\mathrm{B}$ and a higher contrast (relative signal) between bone and brain.

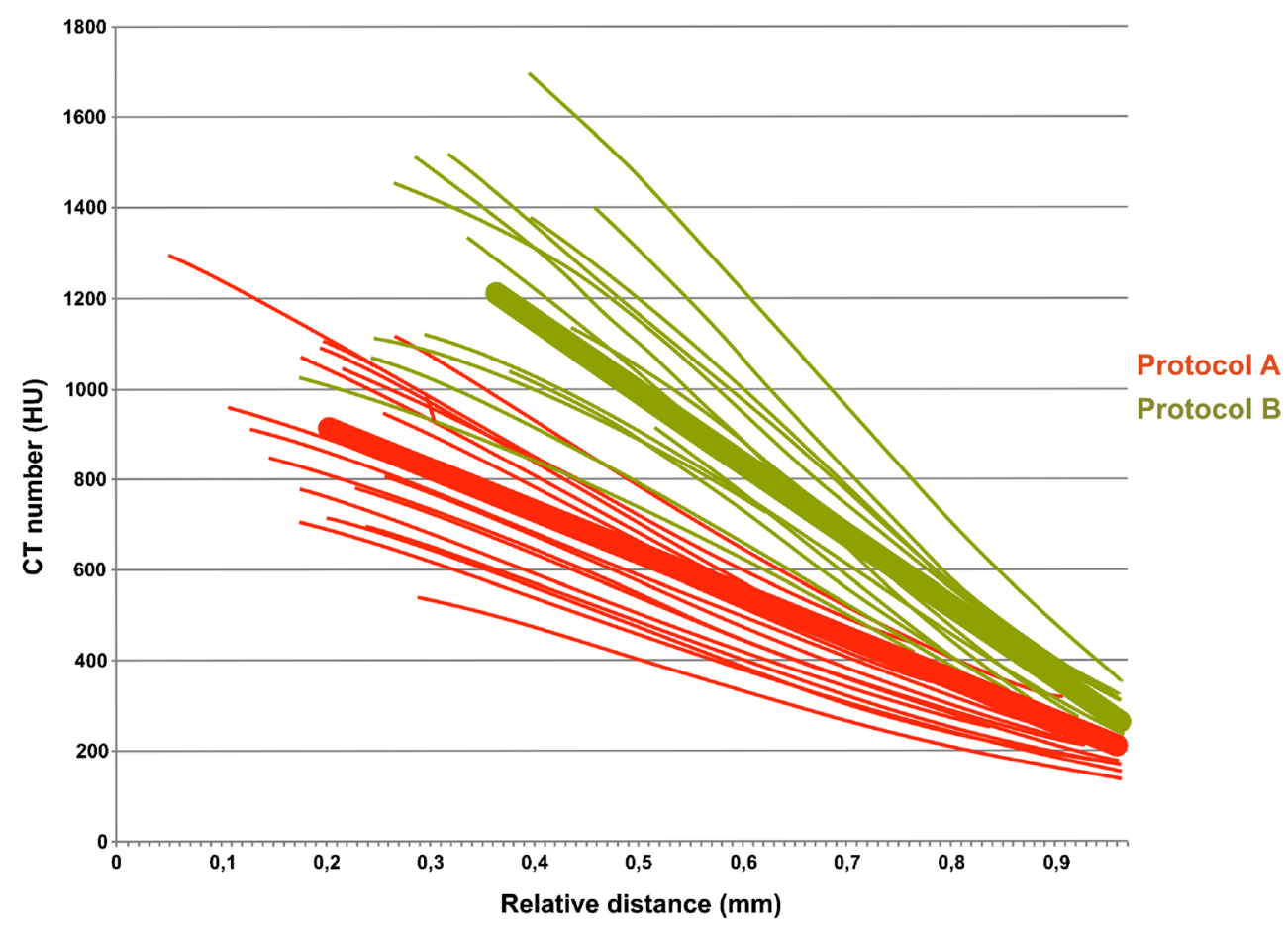


Sharpness of the cranial bone edge was subjectively evaluated as similar in both groups ("well defined with sharp contours"). Objective measurements (the slope of the transition curve bone-brain), however, showed a slightly steeper and, thus, sharper cranial bone edge with protocol B. The difference in slope between the protocols was too small to be subjectively detectable.

The inner ear structures and medial orbital margin were evaluated as less sharp (although still acceptable) with protocol B. However, these structures are not important in the diagnosis of craniosynostosis. In addition, it is accepted that a dedicated high resolution CT is necessary to assess inner ear structures. We emphasize that protocol B is only suitable for evaluation of cranial bones and sutures and not for brain parenchyma or other structures. CT protocols need to be tailored to a specific indication.

When reducing $\mathrm{kVp}$ and/or $\mathrm{mA}$, artefacts including photon starvation and beam hardening increase [27, 28]. The impact of artefacts on diagnostic quality was similar ("minor artefacts" for both protocols despite the major difference in radiation dose ( $97 \%$ dose reduction in protocol B). This is likely related to the use of MBIR, which decreases artefacts in equal dose settings $[17,18]$.

The number and impact of artefacts was similar for both protocols, but the type was different. Beam hardening was present in both groups, but staircase artefacts were only seen on images of protocol B.

Interreader agreement was found to be substantial to almost perfect for both groups.

Some limitations of our work have to be considered. Most importantly, our results only apply to protocol B with the MBIR algorithm and cannot be extrapolated to other types of IR technique with similar dose reductions. Despite efforts to blind the readers to the protocol that was used, they could easily distinguish MBIR from FBP series due their typical image appearance. Also, only the sutural anatomy of the major sutures was analyzed; hence, no information concerning the identification of the skull base sutures ('minor sutures') can be provided [29]. Further, since the therapeutic follow-up of the patients was not included in this study, no information concerning the concordance between radiological evaluation and surgery findings can be given. Finally, our sample size was relatively small.

In summary, with a dose reduction of $97 \%$ to a CTDIvol of $0.94 \mathrm{mGy}$, image quality remained similar in terms of overall diagnostic acceptability, objective noise, subjective cranial bone edge sharpness, and number and impact of artefacts. In addition, protocol $\mathrm{B}$ was superior with regard to objective sharpness of bone-brain interface and subjective noise.

We conclude that, in the diagnosis of craniosynostis in children with cranial deformities, a dedicated sub $0.1 \mathrm{mSv}$ cranial 3DCT protocol can replace the standard protocol with similar to superior image quality.
Acknowledgments The scientific guarantor of this publication is Caroline Ernst. The authors of this manuscript declare relationships with the following companies: GE Healthcare. The authors state that this work has not received any funding. One of the authors has significant statistical expertise. Institutional Review Board approval was obtained. Written informed consent was waived. Methodology: retrospective.

Open Access This article is distributed under the terms of the Creative Commons Attribution-NonCommercial 4.0 International License (http:// creativecommons.org/licenses/by-nc/4.0/), which permits any noncommercial use, distribution, and reproduction in any medium, provided you give appropriate credit to the original author(s) and the source, provide a link to the Creative Commons license, and indicate if changes were made.

\section{References}

1. Vasquez JL, Pombar MA, Pumar JM, del Campo VM (2013) Optimized low-dose multidetector CT protocol for children with cranial deformity. Eur Radiol 23:2279-2287

2. Khanna PC, Thapa MM, Iyer RS, Prasad SS (2011) Pictorial essay: the many faces of craniosynostosis. Indian J Radiol Imaging 21(1): 49-56

3. Badve CA, Mallikarjunappa MK, Iyer RS, Ishak GE, Khanna PC (2013) Craniosynostosis: imaging review and primer on computed tomography. Pediatr Radiol 43(6):728-742, quiz 5-7

4. Frush DP, Applegate KE (2011) 3 radiation risk from medical imaging: a special need to focus on children. Evidence-based imaging: improving the quality of imaging in patient care. Springer, New York, pp 27-41

5. Frush DP, Donnelly LF, Rosen NS (2003) Computed tomography and radiation risks: what pediatric health care providers should know. Pediatrics 112(4):951-957

6. Strauss KJ, Goske MJ, Kaste SC et al (2010) Image gently: ten steps you can take to optimize image quality and lower CT dose for pediatric patients. AJR 194(4):868-873

7. Pearce MS, Salotti JA, Little MP et al (2012) Radiation exposure from CT scans in childhood and subsequent risk of leukaemia and brain tumours: a retrospective cohort study. Lancet 380(9840):499-505

8. Mathews JD, Forsythe AV, Brady Z et al (2013) Cancer risk in 680, 000 people exposed to computed tomography scans in childhood or adolescence: data linkage study of 11 million Australians. BMJ 346:f2360

9. Brenner DJ, Hall EJ (2007) Computed tomography-an increasing source of radiation exposure. NEJM 357(22):2277-2284

10. Brenner D, Elliston C, Hall E, Berdon W (2001) Estimated risks of radiation-induced fatal cancer from pediatric CT. AJR 176(2):289_ 296

11. Paterson A, Frush DP (2007) Dose reduction in paediatric MDCT: general principles. Clin Radiol 62(6):507-517

12. Mulkens T, Salgado R, Bellinck P Dose optimizations and reduction in CT of the brain and head and neck region. Dose reduction in multidetector computed tomography. Berlin, Germany: Springer-Verlag

13. Mieville FA, Berteloot L, Grandjean A et al (2013) Model-based iterative reconstruction in pediatric chest CT: assessment of image quality in a prospective study of children with cystic fibrosis. Pediatr Radiol 43(5):558-567

14. Katsura M, Matsuda I, Akahane M et al (2012) Model-based iterative reconstruction technique for radiation dose reduction in chest $\mathrm{CT}$ : comparison with the adaptive statistical iterative reconstruction technique. Eur Radiol 22(8):1613-1623

15. Neroladaki A, Botsikas D, Boudabbous S, Becker CD, Montet X (2013) Computed tomography of the chest with model-based 
iterative reconstruction using a radiation exposure similar to chest X-ray examination: preliminary observations. Eur Radiol 23(2): 360-366

16. Willemink MJ, de Jong PA, Leiner T et al (2013) Iterative reconstruction techniques for computed tomography part 1: technical principles. Eur Radiol 23(6):1623-1631

17. Deak A, Grimm J, Treitl M et al (2013) Filtered back projection, adaptive statistical iterative reconstruction and a model-based iterative reconstruction in abdominal CT: an experimental clinical study. Radiology 266(1):197-206

18. Yamada Y, Jinzaki M, Tanami Y et al (2012) Model-based iterative reconstruction technique for ultralow-dose computed tomography of the lung: a pilot study. Invest Radiol 47(8):482-489

19. Mieville FA, Gudinchet F, Brunelle F, Bochud FO, Verdun FR (2013) Iterative reconstruction methods in two different MDCT scanners: physical metrics and 4-alternative forced-choice detectability experiments-a phantom approach. Phys Med PM Int J Devoted Appl Phys Med Biol Off J Ital Assoc Biomed Phys 29(1):99-110

20. Korn A, Fenchel M, Bender B et al (2012) Iterative reconstruction in head CT: image quality of routine and low-dose protocols in comparison with standard filtered back-projection. AJNR 33(2):218-224

21. Vorona GA, Zuccoli G, Sutcavage T, Clayton BL, Ceschin RC, Panigrahy A (2013) The use of adaptive statistical iterative reconstruction in pediatric head CT: a feasibility study. AJNR 34(1):205-211
22. Viera AJ, Garrett JM (2005) Understanding interobserver agreement: the kappa statistic. Fam Med 37(5):360-363

23. Craven CM, Naik KS, Blanshard KS (1995) Multispiral three-dimensional computed tomography in the investigation of craniosynostosis: technique optimization. Br J Radiol 68: 724-730

24. Foley SJ, Mcentee MF, Rainford LA (2012) Establishment of CT diagnostic reference levels in Ireland. Br J Radiol 85:1390-1397

25. PiDRL (2014) European Diagnostic Reference Levels for Paediatric Imaging. European Society of Radiology, Austria. Available via http://www.myesr.org/cms/website.php?id=/30447/ en/eu_affairs/esr_subcommittee_on_radiation_protection/ec tender_project_european_diagnostic_reference_levels_for_ paediatric_imaging/pidrl___european_diagnostic_reference_ levels_for_paediatric_imaging.htm. Accessed 20 Jan 2015

26. Applegate KE, Thomas K (2011) Pediatric CT-the challenge of dose records. Pediatr Radiol 41(Suppl 2):523-527

27. Barrett JF, Keat N (2004) Artifacts in CT: recognition and avoidance. Radiographics Rev Publ RSNA, Inc 24(6):1679-1691

28. Boas FE, Fleischmann D (2012) CT artifacts: causes and reduction techniques. Imaging Med 4(2):229-240

29. Calandrelli R, D'Apolito G, Gaudino S (2014) Radiological assessment of skull base changes in children with syndromic craniosynostosis: role of "minor" sutures. Neuroradiology 56:865-875 